\title{
High Throughput Profiling of Antibiotic Resistance Genes in Urban Park Soils with Reclaimed Water Irrigation
}

\author{
Feng-Hua Wang, ${ }^{\dagger, \dagger}$ Min Qiao, ${ }^{\dagger}$ Jian-Qiang Su, ${ }^{\S}$ Zheng Chen, ${ }^{\dagger}$ Xue Zhou, ${ }^{\dagger, \dagger}$ and Yong-Guan Zhu* ${ }^{*},, \S$ \\ ${ }^{\dagger}$ State Key Lab of Urban and Regional Ecology, Research Center for Eco-Environmental Sciences, Chinese Academy of Sciences, \\ Beijing 100085, People's Republic of China \\ ${ }^{\ddagger}$ University of Chinese Academy of Sciences, Beijing 100049, People’s Republic of China \\ ${ }^{\S}$ Key Lab of Urban Environment and Health, Institute of Urban Environment, Chinese Academy of Sciences, Xiamen 361021, \\ People's Republic of China
}

\section{Supporting Information}

ABSTRACT: Reclaimed water irrigation (RWI) in urban environments is becoming popular, due to rapid urbanization and water shortage. The continuous release of residual antibiotics and antibiotic resistance genes (ARGs) from reclaimed water could result in the dissemination of ARGs in the downstream environment. This study provides a comprehensive profile of ARGs in park soils exposed to RWI through a high-throughput quantitative PCR approach. 147 ARGs encoding for resistance to a broad-spectrum of antibiotics were detected among all park soil samples. Aminoglycoside and beta-lactam were the two most dominant types of ARGs, and antibiotic deactivation and efflux pump were the two most dominant mechanisms in these RWI samples. The total enrichment of ARGs varied from 99.3-fold to 8655.3-fold compared to respective controls. Six to 60 ARGs were statistically enriched among these RWI samples. Four transposase genes were detected in RWI samples. TnpA-04 was the most enriched transposase gene with an enrichment was up to 2501.3-fold in Urumqi RWI samples compared with control soil samples. Furthermore, significantly positive correlation was found between ARGs and transposase abundances, indicating that transposase might be involved in the propagation of ARGs. This study demonstrated that RWI resulted in the enrichment of ARGs in urban park soils.

\section{INTRODUCTION}

The propagation of antibiotic resistance genes (ARGs) has become an emerging public health problem, and ARGs are now considered as environmental contaminants. ${ }^{1-3}$ ARGs could be further disseminated among bacteria through horizontal gene transfer mechanisms via mobile genetic elements (MGEs), such as transposons, plasmids, and integrons. ${ }^{4,5}$ The assessment of ARGs reservoirs and persistence in the environment is therefore critical for implementing strategies to mitigate ARGs dissemination. ${ }^{6}$

Numerous studies have demonstrated that wastewater treatment plants (WWTPs) are important reservoirs for ARGs. $^{7-10}$ Antibiotics and ARGs in sewage water enter WWTPs from hospitals, private households, pharmaceutical plant wastewater, and other sources. However, the conventional biological processes in WWTPs cannot eliminate antibiotics and ARGs completely since they are mainly designed for the removal of solids, organic matter, and nutrients. ${ }^{7111,12}$ Gao et al. found that $\operatorname{tet}(\mathrm{O})$, tet $(\mathrm{W})$, and sulI genes were detected at high concentrations of $9.1 \times 10^{3}, 5.1 \times 10^{3}$, and $1.1 \times 10^{4}$ copies/ $\mathrm{mL}$, respectively, in the final effluent of a conventional WWTP. ${ }^{8}$ Even advanced treatment systems in WWTPs were developed to remove organic substrates and nutrients not for ARGs. ' For example, UV processes have failed to reduce
ARGs. ${ }^{13}$ Chen and Zhang detected high concentrations of tet $\left(2.5 \times 10^{4}\right.$ to $1.2 \times 10^{4}$ copies $\left./ \mathrm{ml}\right)$ and sul genes $\left(6.6 \times 10^{5}\right.$ to $3.1 \times 10^{6}$ copies $\left./ \mathrm{mL}\right)$ in the effluent of different WWTPs after advanced treatment process. ${ }^{9}$ Thus, many studies have now illustrated the occurrence of ARGs and antibiotics in wastewater, sludge, and reclaimed water. ${ }^{14-18}$ Due to rapid urbanization and the shortage of water, reclaimed water irrigation (RWI) in urban environment is becoming an important option. ${ }^{19}$ However, the impact of RWI on ARGs in soils remains unclear. ${ }^{20}$ The continuous release of residual antibiotics and ARGs from WWTPs effluent could result in the propagation and dissemination of ARGs in the downstream environment. ${ }^{11,20,21}$ Furthermore, the propagation and dissemination of ARGs could be facilitated via horizontal gene transfer mechanisms. ${ }^{22}$ Reclaimed water is evidently a reservoir of $\mathrm{ARGs}^{23}$ and, therefore, the effect of irrigation with reclaimed water on ARGs needs definition.

The availability of primers is one of the technical obstacles in assessing the diversity and abundance of ARGs. Several studies

Received: May 29, 2014

Revised: July 23, 2014

Accepted: July 24, 2014

Published: July 24, 2014 
have used PCR and quantitative PCR ( $\mathrm{qPCR}$ ) to determine the presence of ARGs in irrigated soils. ${ }^{19,20,23}$ These approaches only target a few well-studied resistance genes, ${ }^{7,20}$ and are not applicable for broad-spectrum scanning of ARGs in environmental samples. ${ }^{13}$ In the present study, a high-throughput qPCR with 295 validated primer sets (including 285 ARGs, 9 transposase genes, and 16S rRNA gene) has been used to investigate the impact of RWI on ARGs in public park soils of representative Chinese cities. Since ARGs can be present naturally, ${ }^{24,25}$ the background level needs to be considered. So, soil samples without using reclaimed water were also collected as control for each RWI park soil.

\section{MATERIALS AND METHODS}

Site Selection and Sampling. Soil samples from eight representative public parks, which irrigated with reclaimed water from respective local WWTPs, were collected in 2012 from seven Chinese cities including Beijing (two Beijing parks, referred to as BJT1, BJT2), Shijiazhuang (SJZT), Jinan (JNT), Wuhan (WHT), Kunming (KMT), Baotou (BTT) and Urumqi (WQT) (Supporting Information (SI) Figure S1). Control soil samples were collected from public parks without using reclaimed water from respective cities including Beijing (named BJC), Jinan (JNC), Wuhan (WHC), Kunming (KMC), Baotou (BTC) and Urumqi (WQC). The samples from Shijiazhuang and Beijing shared the same control (BJC) because they are geographically close. Detailed information on the sampling sites is presented in SI Table S1. At each site, the upper $10 \mathrm{~cm}$ of soil was collected from four plots. All samples were transported to the laboratory protected with dry ice and stored at $-80{ }^{\circ} \mathrm{C}$ before DNA extraction and chemical analysis.

DNA Extraction. High-molecular-weight community DNA was extracted by the freeze-grinding, SDS-based method ${ }^{26}$ and was purified using a low-melting agarose gel followed by phenol extraction. The quality and concentration of the DNA were determined using $1.2 \%$ agarose gel electrophoresis and a spectrophotometer analysis (NanoDrop ND-1000, NanoDrop Technologies, Willmington, DE).

Primer Design. The primer design followed methods described by Zhu et al. ${ }^{27}$ and Stedtfeld et al. ${ }^{28}$ For this study, 295 validated primer sets were used, including 285 ARGs for all major classes of ARGs, 9 transposase genes, and 1 16S rRNA gene (SI Table S2).

Antibiotic Analyses. The extraction procedures for tetracyclines, quinolones, sulfonamides, and their degradation products in soils followed methods described before with some modification. ${ }^{19}$ LC-MS/MS was used to separate and detect the antibiotics as described by Zhang et al. ${ }^{29}$ Five target tetracyclines, including tetracycline (TC), oxytetracycline (OTC), chlortetracycline (CTC), methacycline (MTC), and doxycycline (DXC), and nine degradation products, including anhydrotetracycline (ATC), 4-epitetracycline (ETC), 4-epianhydrotetracycline (EATC), 4-epioxytetracycline (EOTC), $\alpha$ apo-oxytetracycline ( $\alpha$-apo-OTC), $\beta$-apo-oxytetracycline $(\beta$ apo-OTC), isochlortetracycline (ICTC), 4-epianhydrochlortetracycline (EACTC), demethylchlortetracycline (DMCTC), four sulfonamides, including sulfadimethoxine (SDM), sulfamerazine (SMI), sulfamethizole (SMT), sulfamethoxazole (SMZ), and six fluoroquinolones, including ofloxacin (OFL), enrofloxacin (ENRO), sarafloxacin (SARA), danofloxacin (DANO), ciprofloxacin (CIP), norfloxacin (NORF), were analyzed in this study.
Quantitative PCR. All high-throughput qPCR reactions were performed using the Wafergen SmartChip Real-time PCR system. This SmartChip nanowell platform can be used for large scale gene expression studies, which can process 5,184 nanowell reactions per run. Quality assurance/quality control (QA/QC) was performed according to a standard protocol provided by Wafergen Biosystems. PCR mixtures (100 nl for each well) consisted of 1 X LightCycler 480 SYBR Green I Master (Roche Applied Sciences, Indianapolis, IN), $1 \mathrm{mg} / \mathrm{mL}$ bovine serum albumin (New England Biolaboratories, Beverly, $\mathrm{MA}$ ), $500 \mathrm{nM}$ each primer and a DNA template of $5 \mathrm{ng} / \mu \mathrm{L}$. PCR mixtures were dispensed into a 5184-wells chip using SmartChip Multisample Nanodispenser (MSND) in 296 (assays) $\times 16$ (samples) format, followed by real-time qPCR using SmartChip Cycler. A nontemplate control was included on each chip for each primer set. After the initial enzyme activation at $95{ }^{\circ} \mathrm{C}$ for $10 \mathrm{~min}, 40$ cycles of the following program were used for amplification: denaturation at $95{ }^{\circ} \mathrm{C}$ for $30 \mathrm{~s}$, annealing at $60{ }^{\circ} \mathrm{C}$ for $30 \mathrm{~s}$. The melting process was automatically generated by Wafergen software. qPCR results were analyzed using SmartChip qPCR Software (v 2.7.0.1), wells with multiple melting peak as well as wells with amplification efficiency beyond the range (1.8-2.2) were discarded. All qPCR reactions were performed in triplicate and a threshold cycle $(\mathrm{Ct})$ of 31 was used as the detection limit. Only samples with three or four replicates which had amplification were regarded as positive. In the calculation of the $\Delta \mathrm{Ct}$ of the reference sample, if there were no amplification, the detection limit $\mathrm{Ct}$ (31) was used. Genes were considered statistically enriched if the range created by two standard deviations of the mean fold change was entirely $>1 .^{27,28}$

Averages, standard deviations, and fold change values of all data were determined using Microsoft Excel 2010. Pearson correlations and box plots were performed by SPSS13.0. Circos graphs were produced via Circos software (http://circos.ca/) on line. Heatmap graphs were produced using RStudio with gplots package.

\section{RESULTS AND DISCUSSION}

Diversity of Antibiotic Resistance Genes. A total of 147 ARGs were detected among all of the samples (SI Figure S2). An average of 87 ARGs was detected among these eight RWI park soils. Up to 113 ARGs were detected in BTT samples, while only 40 ARGs were detected in the BJT2 sample, suggesting that the occurrence of ARGs varied among different RWI samples. The high detection frequencies of ARGs indicated that ARGs existed widely in RWI soils. Even for the six control samples, an average of 81 ARGs were detected, especially in the BTC sample, where up to 106 ARGs were detected, demonstrating that ARGs are diverse and ubiquitous in natural environments. ${ }^{25}$ Previous studies have demonstrated that antibiotic resistance is a natural and ancient phenomenon and ARGs have been found in permafrost sediments, ${ }^{25}$ pristine forest, ${ }^{27}$ and deep oceans. ${ }^{30}$

Judged for the 147 ARGs detected, antibiotic deactivation (41\%) and efflux pump (39\%) were the two most dominant resistance mechanisms, followed by cellular protection resistance mechanism (18\%) (Figure 1A). The diverse set of resistance genes detected could potentially confer resistance to all the major classes of antibiotics, including antibiotics critically important for human medicine, ${ }^{27}$ such as aminoglycosides, beta-lactam, macrolides, and vancomycin (Figure 1B). Vancomycin is recognized as a "last-resort" life-saving anti- 


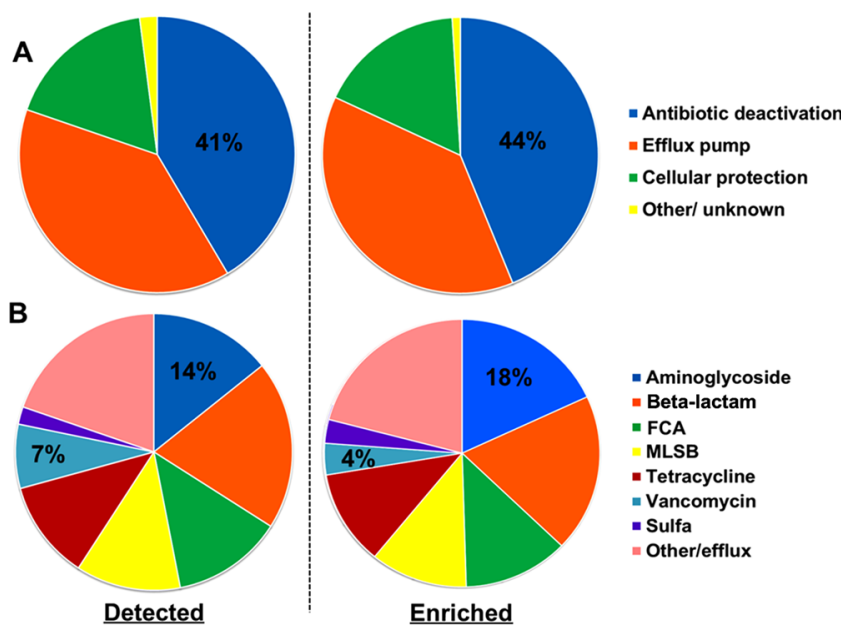

Figure 1. Resistance genes detected in all samples were classified based on (A) the mechanism of resistance, and (B) the antibiotic to which they confer resistance. FCA, fluoroquinolone, quinolone, florfenicol, chloramphenicol, and amphenicol resistance genes; MLSB, Macrolide-Lincosamide-Streptogramin B resistance.

biotic. $^{31}$ The distribution of ARG type in each RWI sample is shown in Figure 2. Eight types of ARGs were detected in RWI

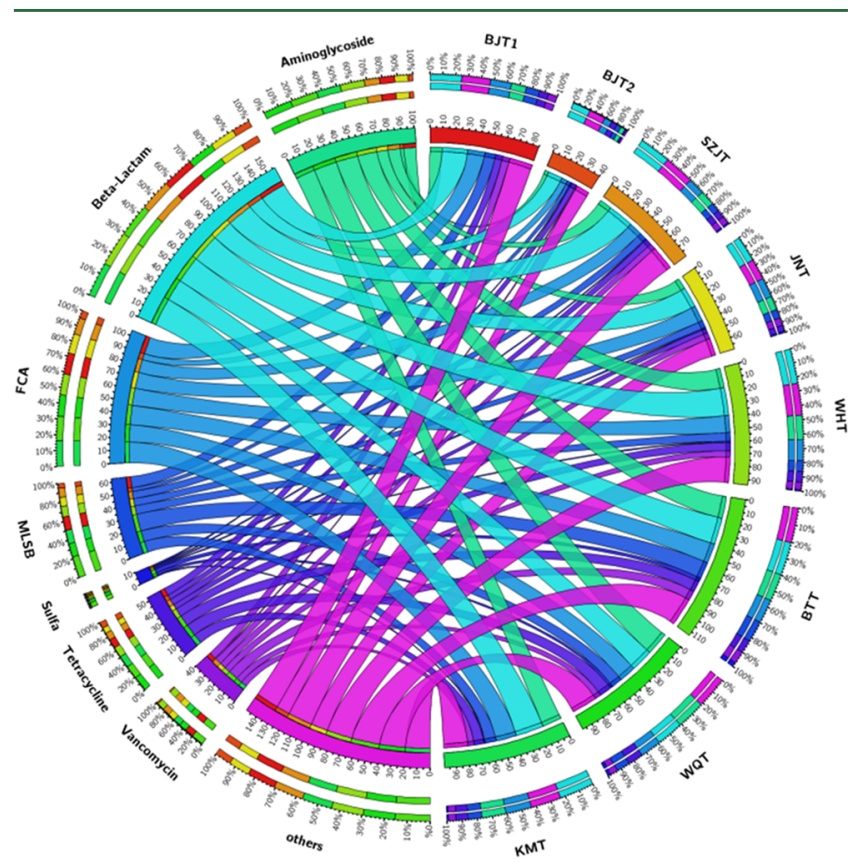

Figure 2. Distribution of each ARGs type in eight park soils with reclaimed water irrigation (RWI). The data were visualized via Circos software (http://circos.ca/). The length of the bars of each sample on the outer-ring represented the percentage of ARGs in each sample.

samples. Resistance genes for beta-lactam (18.4-30.0\%), FCA (fluoroquinolone, quinolone, florfenicol, chloramphenicol, and amphenicol) (12.5-22.0\%), and aminoglycoside (7.5-19.4\%) were the three most dominant types in RWI samples, followed by resistance genes for MLSB (Macrolide-LincosamideStreptogramin) (7.4-12.4\%), tetracycline (6.3-10.2\%), vancomycin $(2.5-8.5 \%)$, and sulfa (ND (not detected) 2.7\%). The distribution of ARGs was quite different among these RWI samples. The data showed the existence of a broad-spectrum of different ARGs, some of which have never been reported in irrigated park soil samples before. ${ }^{19}$ Such broad-spectrum distribution of different types of ARGs in RWI samples was quite different from that in activated sludge samples. ${ }^{11,32}$ Previous studies have found that the most abundant ARGs in genomic metagenome and plasmid metagenome were aminoglycoside resistance genes and tetracycline resistance genes, respectively. ${ }^{11,32}$

Abundance of Antibiotic Resistance Genes in RWI Samples. Resistance gene profiles indicated the patterns and degrees of enrichment of ARGs for each RWI sample (Figure 3 ). We used the sum of the enrichment of all ARGs in a sample

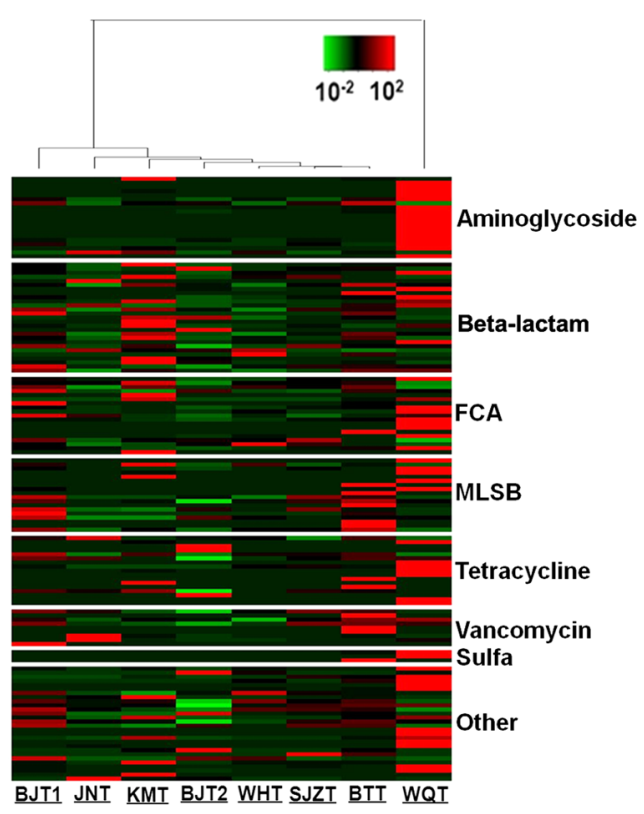

Figure 3. Resistance gene profile from eight park soils with reclaimed water irrigation (RWI). Each column is labeled with the sample name, and each row is the results from a single primer set. Resistance profiles that confer resistance to all major classes of antibiotics as follows: aminoglycoside, beta-lactam, FCA, MLSB, tetracycline, vancomycin, sulfa, and others.

to approximate total enrichment in RWI samples. The total enrichment of ARGs varied from 99.3-fold (SJZT) to 8655.3fold (WQT) compared to respective controls, demonstrating the large discrepancy of the antibiotic resistance in these RWI samples. It was obvious that the trends in variation for each antibiotic type were different among RWI samples, and the maximum abundance for each antibiotic type occurred mostly in WQT sample, except for beta-lactam and vancomycin. Taken together with the results of fold change for each subtype of ARG (SI Table S4), the total abundance of aminoglycoside resistance genes was up to 3996.7-fold with strB being the highest enriched gene (805.6-fold) in WQT samples compared with WQC samples. In contrast, only a 5.6-fold change was found in BJT2 samples compared with BJC samples. For FCA resistance genes, the total abundance was from 4.4-fold (BJT2) to 365.2-fold (WQT). For tetracycline resistance genes, the total abundance was 763.9-fold in WQT samples, with tet $(\mathrm{X})$ (338.5-fold) and tet(G-02) (308.9-fold) were the two most abundant genes compared with WQC samples. In comparison, no more than 5.0-fold was found in SJZT, JNT, and WHT samples compared with their respective control soil samples. The fold change of sulII gene encoding for sulfa resistance was up to 1173.7-fold in WQT samples, while the total enrichment 
of sulfa resistance genes was 1243.4-fold compared with WQC samples. The maximum abundance of beta-lactam resistance genes was 124.1-fold in KMT sample compared with the KMC sample. For vancomycin resistance genes, vanYD-02 had the largest abundance in BJT1 samples (192.1-fold) compared with BJC samples. All these ARGs with the higher abundance have been frequently detected in WWTPs. ${ }^{18,21,33,34}$ We found that the enrichment of ARGs was much higher in WQT than in other RWI samples, which might be caused by the higher abundances of residual antibiotics and ARGs in WWTPs, as WWTPs are considered important reservoirs of ARGs. ${ }^{15,26,34}$ Other factors could also result in this phenomenon, such as different irrigation histories (SI Table S1), irrigation volume, as well as the native abundance of ARGs in soil. ${ }^{19,24}$ Although the abundances of some ARGs in this study were less than previous studies, ${ }^{27,35}$ the findings could still demonstrate that the continuous release of residual ARGs and antibiotics from WWTPs effluent could result in the dissemination of ARGs in the downstream environment. ${ }^{21}$

Statistical Enrichment of Antibiotic Resistance Genes in RWI Samples. Among all the detected 147 ARGs, a total of 105 unique ARGs were significantly enriched in the RWI samples. Six to 60 ARGs were significantly enriched among these eight RWI samples (Figure 4). In addition, the total

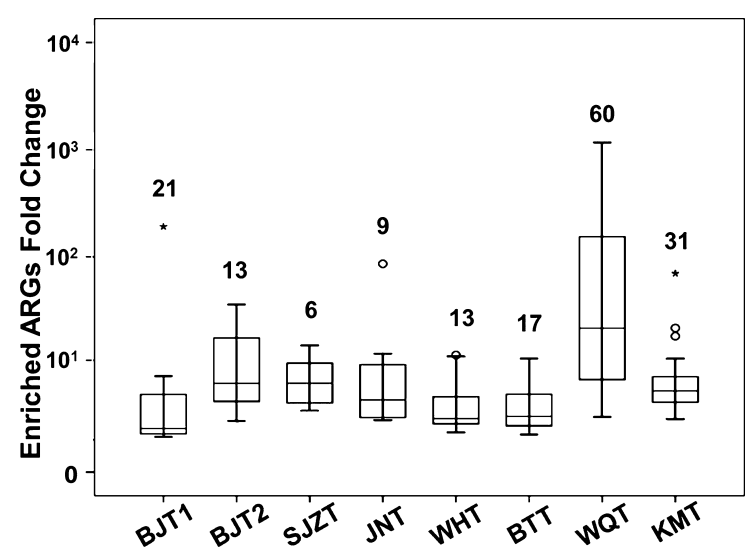

Figure 4. Statistically enriched resistance genes are showed in the box plots. The number above each site indicates the number of primer sets that yielded statistically significant results. The symbols indicate the following: box 25th to 75th percentile; horizontal line, median; whiskers, 10th and 90th percentile; and square, maximum value. The $y$ axis is a log scale of fold change.

enrichment of ARGs had distinct differences ranging from 41.1fold (SJZT) to 8623.1-fold (WQT) compared with their respective soil controls. It was also found that different ARGs had various extents of enrichment for each RWI sample. For example, the maximum enrichment of a single ARG was up to 1173.7-fold (sulII) in the WQT sample compared to the corresponding WQC. Other genes such as qacEdeltal-02 and strB were enriched over 800.0-fold compared with the control soil. The minimum enrichment of a single ARG was only 2.3fold $\left(\operatorname{aac}\left(6^{\prime}\right) L 1\right)$ in the WQT sample. Although not all ARGs had higher enrichment, the findings suggest that RWI partly enrich certain ARGs in urban park soils.

From the detected profile to the enriched profile, the percentage of antibiotic deactivation mechanism increased from $41 \%$ to $44 \%$, while other mechanisms remained almost the same $( \pm 1 \%)$ (Figure $1 \mathrm{~A})$. From Figure $1 \mathrm{~B}$, it can be seen that the percentage of resistance conferred on aminoglycosides was increased from $14 \%$ (detected) to $18 \%$ (enriched). The percentage of resistance for vancomycin was decreased from $7 \%$ (detected) to $4 \%$ (enriched). Thus, aminoglycoside resistance genes were more easily enriched than other types of ARGs, which might be related to the common use of aminoglycosides. ${ }^{15,27}$ Previous studies demonstrate that treated wastewater irrigation results in fluctuations of bacterial phyla, which further cause the fluctuations in the relative abundance of different ARG families. ${ }^{36}$ SI Figure S3 illustrates the profile of statistically enriched ARGs in each RWI sample. Comparing the detected profile to the enriched profile, the profiles of detected genes in each RWI sample were quite similar, whereas the profiles of genes enriched were much more variable, indicating a specific type of ARGs was selected in each RWI sample. For example, the sulfa resistance genes were highly enriched in SJZT, JNT, and BTT samples. At the same time, the MLSB and tetracycline resistance genes were highly enriched in BJT1 and BJT2 samples, respectively. The percentage of different mechanisms was also different in each RWI sample between the detected and enriched profiles. With the high-throughput qPCR assay, we were able to profile the impact of RWI on ARGs in park soils more comprehensively.

Previous studies have investigated the role of WWTPs in the dissemination and proliferation of ARGs in various environments using PCR and conventional qPCR approaches. ${ }^{20,21,37,38}$ But these approaches have not been amenable to broadspectrum scanning of ARGs in environmental samples. ${ }^{13}$ In a recent study on the effect of treated wastewater irrigation on the magnitude of ARGs, six different ARGs (qnrA, tet (O), sulI, sulII, ermB, and ermF) were analyzed using the conventional qPCR method. ${ }^{20}$ It was found that treated wastewater irrigation could not enrich ARGs. ${ }^{20}$ Usefully, the molecular signature of ARGs can be served as a suitable marker to indicate the degree of anthropogenic impact. ${ }^{30,39,40}$ High-throughput qPCR profiling of ARGs is an essential way to characterize the molecular trait of ARGs in different RWI soils since it can provide broader profiles of ARGs and allow estimation of anthropogenesis.

Correlation between ARGs and Transposase Abundances. Among the nine transposase genes, four were detected in at least one RWI sample (Figure 5A). TnpA-02 gene was detected in all RWI samples with an average enrichment of 1.8fold compared with their respective soil controls. The total transposase gene enrichment was up to 2958.6-fold (Figure $5 \mathrm{~A})$. The total abundance of transposase genes was correlated (Figure 5B) with the total abundances of tetracycline and sulfa resistance genes, respectively (SI Table $S 6, P<0.01$ ). Previous studies have found that tetracycline resistance genes, such as tet $(\mathrm{M})$, tet $(\mathrm{W})$, and tet $(\mathrm{Q})$, are always present in transposons and other MGEs. ${ }^{41-43}$ The sulII gene is found to be located on four IncN plasmids in WWTPs effluent, ${ }^{44}$ and these IncN plasmids have also been detected in clinical samples, and regarded as having human health relevance. A plasmid metagenome analysis from the final effluent of a WWTP in Germany has revealed 140 clinically relevant ARGs including genes encoding resistance to aminoglycosides, beta-lactams, fluoroquinolones, macrolides, tetracyclines, and other antibiotics. ${ }^{37}$ Previous study has also confirmed that E. coli, K. pneumoniae, and P. mirabilis harbor plasmid-mediated AmpC enzymes. ${ }^{45}$ In the present study, floR gene encoding for FCA resistance (152.7-fold) was highly enriched in the WQT sample compared with WQC sample. The floR gene has been found to be part of the tnfloR transposon from Escherichia coli. ${ }^{46}$ For 


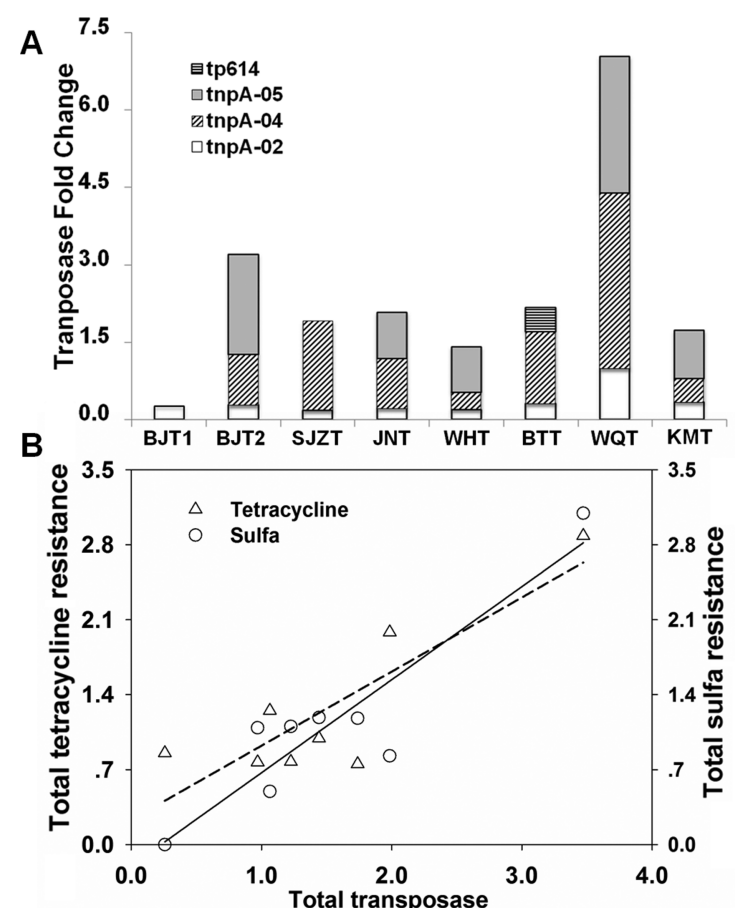

Figure 5. Abundance of transposase (A) and correlations of total tetracycline/sulfa resistance with transposase abundance (B). The data used was logarithmic transformation. $P<0.01$ indicates the significant correlation.

MLSB resistance genes, ermB gene was enriched 126.5-fold (compared with control soil sample), and $\mathrm{ermB}$ gene has been found on transposons, such as Tn917 and Tn1545. ${ }^{47}$ Plainly, those ARGs which are associated with MGEs can move from one species to another through horizontal gene tranfer mechanisms, ${ }^{34}$ with easy dissemination and obstinate persistance in various environments.

RWI can introduce transposons or other MGEs harboring ARGs to the soil microbiome, and the high mobility and versatility of these elements may contribute to ARGs propagation among many native soil bacteria. ${ }^{37}$ In our study, we have observed significantly positive correlations between ARGs and transposase abundance, indicating the potential facilitation of ARGs transfer. Therefore, when considering the impact of RWI, it is necessary not only to assess the abundances of ARGs in RWI soils but also to investigate the abundances of MGEs.

Correlation between ARGs Abundances and Antibiotic Concentrations. SI Figure S4A shows that the total tetracycline concentrations in RWI samples were higher than their controls (except BTT). The total tetracycline concentrations ranged from $4.0 \mu \mathrm{g} \mathrm{kg}^{-1}$ to $68.9 \mu \mathrm{g} \mathrm{kg}^{-1}$, while for controls, the total concentrations were in the range of $\mathrm{ND} \sim$ $28.0 \mu \mathrm{g} \mathrm{kg}^{-1}$. For the six quinolones, enrofloxacin and ofloxacin were the most observed in RWI samples (SI Figure S4B). The highest mean concentrations of enrofloxacin $\left(181.3 \mu \mathrm{g} \mathrm{kg}^{-1}\right)$ and ofloxacin $\left(89.0 \mu \mathrm{g} \mathrm{kg}^{-1}\right)$ were observed in WQT samples, while for controls, the highest total quinolone concentration was only $2.2 \mu \mathrm{g} \mathrm{kg}^{-1}$ in the KMC sample. None of the four sulfonamides (SMZ, SLM, SMI, and SMT) were detected in any soil samples. Our data indicate the potential for antibiotic residues to accumulate in soils during RWI, and that they are not fully removed with wastewater treatment. ${ }^{8,22,34}$ The relationships between the abundance of detected ARGs and antibiotic concentrations are shown in SI Table S6. It appears that total tetracyclines were positively correlated not only with the abundance of tetracycline resistance genes, but also with other antibiotic resistance genes, such as those for aminoglycosides, FCA, and sulfa resistance. The concentrations of antibiotics found in this study were much lower than in swine manures, ${ }^{27}$ or farmlands applied with manures, ${ }^{27,48}$ or in soil directly irrigated with wastewater. ${ }^{36}$ However, it has previously been observed that low residual antibiotic contamination could exert selective pressure on ARG induction and/or stimulate horizontal gene transfer of ARGs under certain conditions. ${ }^{39,49,50}$ Furthermore, reservoirs of ARGs have been shown to be stable in bacterial communities, even in the absence of antibiotics. ${ }^{51,52}$ This could explain the high abundance of the sulII gene (1173.7-fold) in the absence of sulfa antibiotics in the RWI sample.

In summary, this study clearly demonstrates the impact of RWI on the enrichment of ARGs encoding for many types of antibiotics such as aminoglycosides and sulfa drugs. The higher diversity and abundance of ARGs in RWI samples than in controls indicates that reclaimed water from WWTPs could be an important source of ARGs. Further studies are needed to investigate the profile of ARGs at different stages of WWTPs with reference to influent and effluent water, which could be achieved using the present high-throughput qPCR method. It is important to identify which specific ARGs could be target genes, and which could help to monitor the quality of reclaimed water in order to reduce the potential threat to human health.

\section{ASSOCIATED CONTENT}

\section{S Supporting Information}

Table S1-S6 and Figures S1-S4. This material is available free of charge via the Internet at http://pubs.acs.org.

\section{AUTHOR INFORMATION}

\section{Corresponding Author}

*Phone: +86-10-62-62936940; fax: +86-10-62936940; e-mail: ygzhu@rcees.ac.cn.

\section{Notes}

The authors declare no competing financial interest.

\section{ACKNOWLEDGMENTS}

We thank Dr. James M. Tiedje and Dr. Robert D. Stedtfeld from Michigan State University for technical assistance. This work is financially supported by Natural Science Foundation of China (No.21210008).

\section{REFERENCES}

(1) Stoll, C.; Sidhu, J. P.; Tiehm, A.; Toze, S. Prevalence of clinically relevant antibiotic resistance genes in surface water samples collected from Germany and Australia. Environ. Sci. Technol. 2012, 46 (17), 9716-9726.

(2) Pruden, A.; Pei, R. T.; Storteboom, H.; Carlson, K. H. Antibiotic resistance genes as emerging contaminants: Studies in northern Colorado. Environ. Sci. Technol. 2006, 40 (23), 7445-7450.

(3) Cooper, M. A.; Shlaes, D. Fix the antibiotics pipeline. Nature 2011, 472, 32.

(4) Gogarten, J. P.; Townsend, J. P. Horizontal gene transfer, genome innovation and evolution. Nat. Rev. Microbiol. 2005, 3 (9), 679-687.

(5) Schluter, A.; Szczepanowski, R; Puhler, A.; Top, E. M. Genomics of IncP-1 antibiotic resistance plasmids isolated from wastewater treatment plants provides evidence for a widely accessible drug resistance gene pool. FEMS Microbiol. Rev. 2007, 31 (4), 449-477. 
(6) Mao, D.; Luo, Y.; Mathieu, J.; Wang, Q.; Feng, L.; Mu, Q.; Feng, C.; Alvarez, P. J. Persistence of extracellular DNA in river sediment facilitates antibiotic resistance gene propagation. Environ. Sci. Technol. 2014, 48 (1), 71-78.

(7) Pruden, A. Balancing water sustainability and public health goals in the face of growing concerns about antibiotic resistance. Environ. Sci. Technol. 2014, 48 (1), 5-14.

(8) Gao, P.; Munir, M.; Xagoraraki, I. Correlation of tetracycline and sulfonamide antibiotics with corresponding resistance genes and resistant bacteria in a conventional municipal wastewater treatment plant. Sci. Total Environ. 2012, 421-422, 173-183.

(9) Chen, H.; Zhang, M. Effects of advanced treatment systems on the removal of antibiotic resistance genes in wastewater treatment plants from Hangzhou, China. Environ. Sci. Technol. 2013, 47 (15), $8157-8163$.

(10) Zhang, X. X.; Zhang, T. Occurrence, abundance, and diversity of tetracycline resistance genes in 15 sewage treatment plants across China and other global locations. Environ. Sci. Technol. 2011, 45 (7), 2598-2604.

(11) Yang, Y.; Li, B.; Ju, F.; Zhang, T. Exploring variation of antibiotic resistance genes in activated sludge over a four-year period through a metagenomic approach. Environ. Sci. Technol. 2013, 47 (18), 10197-10205.

(12) Rizzo, L.; Manaia, C.; Merlin, C.; Schwartz, T.; Dagot, C.; Ploy, M. C.; Michael, I.; Fatta-Kassinos, D. Urban wastewater treatment plants as hotspots for antibiotic resistant bacteria and genes spread into the environment: A review. Sci. Total Environ. 2013, 447, 345360.

(13) Munir, M.; Wong, K.; Xagoraraki, I. Release of antibiotic resistant bacteria and genes in the effluent and biosolids of five wastewater utilities in Michigan. Water Res. 2011, 45 (2), 681-693.

(14) Li, B.; Zhang, T. Mass flows and removal of antibiotics in two municipal wastewater treatment plants. Chemosphere 2011, 83 (9), 1284-1289.

(15) Auerbach, E. A.; Seyfried, E. E.; McMahon, K. D. Tetracycline resistance genes in activated sludge wastewater treatment plants. Water Res. 2007, 41 (5), 1143-1151.

(16) Li, B.; Zhang, T. Biodegradation and adsorption of antibiotics in the activated sludge process. Environ. Sci. Technol. 2010, 44 (9), 34683473.

(17) Zhang, T.; Zhang, M.; Zhang, X. X.; Fang, H. P. H. Tetracycline resistance genes and tetracycline resistance lactose-fermenting Enterobacteriaceae in activated sludge of sewage treatment plant. Environ. Sci. Technol. 2009, 43 (10), 3455-3460.

(18) Szczepanowski, R.; Linke, B.; Krahn, I.; Gartemann, K. H.; Gützkow, T.; Eichler, W.; Pühler, A.; Schlüter, A. Detection of 140 clinically relevant antibiotic-resistance genes in the plasmid metagenome of wastewater treatment plant bacteria showing reduced susceptibility to selected antibiotics. Microbiology 2009, 155 (7), 2306-2319.

(19) Wang, F. H.; Qiao, M.; Lv, Z. E.; Guo, G. X.; Jia, Y.; Su, Y. H.; $\mathrm{Zhu}, \mathrm{Y}$. G. Impact of reclaimed water irrigation on antibiotic resistance in public parks, Beijing, China. Environ. Pollut. 2014, 184, 247-253.

(20) Negreanu, Y.; Pasternak, Z.; Jurkevitch, E.; Cytryn, E. Impact of treated wastewater irrigation on antibiotic resistance in agricultural soils. Environ. Sci. Technol. 2012, 46 (9), 4800-4808.

(21) Czekalski, N.; Díez, E. G.; Bürgmann, H. Wastewater as a point source of antibiotic-resistance genes in the sediment of a freshwater lake. ISME J. 2014, DOI: 10.1038/ismej.2014.8.

(22) Zhang, T.; Li, B. Occurrence, transformation, and fate of antibiotics in municipal wastewater treatment plants. Crit. Rev. Environ. Sci. Technol. 2011, 41 (11), 951-998.

(23) Fahrenfeld, N.; Ma, Y.; O’Brien, M.; Pruden, A. Reclaimed water as a reservoir of antibiotic resistance genes: Distribution system and irrigation implications. Front. Microbiol. 2013, 4, 1-10.

(24) Allen, H. K.; Moe, L. A.; Rodbumrer, J.; Gaarder, A.; Handelsman, J. Functional metagenomics reveals diverse betalactamases in a remote Alaskan soil. ISME J. 2009, 3 (2), 243-251.
(25) D’Costa, V. M.; King, C. E.; Kalan, L.; Morar, M.; Sung, W. W.; Schwarz, C.; Froese, D.; Zazula, G.; Calmels, F.; Debruyne, R.; Golding, G. B.; Poinar, H. N.; Wright, G. D. Antibiotic resistance is ancient. Nature 2011, 477 (7365), 457-461.

(26) Zhou, J. Z.; Bruns, M. A.; Tiedje, J. M. DNA recovery from soils of diverse composition. Appl. Environ. Microbiol. 1996, 62 (2), 316322.

(27) Zhu, Y. G.; Johnson, T. A.; Su, J. Q.; Qiao, M.; Guo, G. X.; Stedtfeld, R. D.; Hashsham, S. A.; Tiedje, J. M. Diverse and abundant antibiotic resistance genes in Chinese swine farms. PNAS 2013, 110 (9), 3435-3440.

(28) Stedtfeld, R. D.; Baushke, S. W.; Tourlousse, D. M.; Miller, S. M.; Stedtfeld, T. M.; Gulari, E.; Tiedje, J. M.; Hashsham, S. A. Development and experimental validation of a predictive threshold cycle equation for quantification of virulence and marker genes by high-throughput nanoliter-volume PCR on the openarray platform. Appl. Environ. Microbiol. 2011, 74 (12), 3831-3838.

(29) Zhang, D. D.; Lin, L. F.; Luo, Z. X.; Yan, C. Z.; Zhang, X. Occurrence of selected antibiotics in Jiulongjiang River in various season, South China. J. Environ. Monit. 2011, 13 (7), 1953-1960.

(30) Chen, B.; Yang, Y.; Liang, X.; Yu, K.; Zhang, T.; Li, X. Metagenomic profiles of antibiotic resistance genes (ARGs) between human impacted estuary and deep ocean sediments. Environ. Sci. Technol. 2013, 47 (22), 12753-12760.

(31) Ziglam, H. M.; Finch, R. G. Limitations of presently available glycopeptides in the treatment of Gram-positive infection. Clin. Microbiol. Infect. 2001, 7, 53-65.

(32) Zhang, T.; Zhang, X. X.; Ye, L. Plasmid metagenome reveals high levels of antibiotic resistance genes and mobile genetic elements in activated sludge. PloS ONE 2011, 6 (10), e26041.

(33) Enne, V. I.; Livermore, D. M.; Stephens, P.; Hall, L. M. C. Persistence of sulphonamide resistance in Escherichia coli in the UK despite national prescribing restriction. Lancet 2001, 357 (9265), $1325-1328$.

(34) Chen, H.; Zhang, M. Occurrence and removal of antibiotic resistance genes in municipal wastewater and rural domestic sewage treatment systems in eastern China. Environ. Int. 2013, 55, 9-14.

(35) Peak, N.; Knapp, C. W.; Yang, R. K.; Hanfelt, M. M.; Smith, M. S.; Aga, D. S.; Graham, D. W. Abundance of six tetracycline resistance genes in wastewater lagoons at cattle feedlots with different antibiotic use strategies. Environ. Microbiol. 2007, 9 (1), 143-151.

(36) Frenk, S.; Hadar, Y.; Minz, D. Resilience of soil bacterial community to irrigation with water of different qualities under Mediterranean climate. Environ. Microbiol. 2014, 16 (2), 559-569.

(37) Gatica, J.; Cytryn, E. Impact of treated wastewater irrigation on antibiotic resistance in the soil microbiome. Environ. Sci. Pollut. Res. 2013, 20 (6), 3529-3538.

(38) Shi, Y. L.; Gao, L. H.; Li, W. H.; Liu, J. M.; Cai, Y. Q. Investigation of fluoroquinolones, sulfonamides and macrolides in long-term wastewater irrigation soil in Tianjin, China. Bull. Environ. Contam. Toxicol. 2012, 89 (4), 857-861.

(39) Storteboom, H.; Arabi, M.; Jessica, G. D.; Pruden, A. Tracking antibiotic resistance genes in the South Platte River basin using molecular signatures of urban, agricultural, and pristine sources. Environ. Sci. Technol. 2010, 44 (19), 7397-7404.

(40) Chen, B. W.; Liang, X. M.; Huang, X. P.; Zhang, T.; Li, X. D. Differentiating anthropogenic impacts on ARGs in the Pearl River Estuary by using suitable gene indicators. Water Res. 2013, 47 (8), $2811-2820$.

(41) Rizzotti, L.; Gioia, F. L.; Dellaglio, F.; Torriani, S. Molecular diversity and transferability of the tetracycline resistance gene tet $(\mathrm{M})$, carried on Tn916-1545 family transposons, in enterococci from a total food chain. Antonie Van Leeuwenhoek 2009, 96 (1), 43-52.

(42) Chopra, I.; Roberts, M. Tetracycline antibiotics: Mode of action, applications, molecular biology, and epidemiology of bacterial resistance. Microbiol. Mol. Biol. Rev. 2001, 65 (2), 232-260.

(43) Kazimierczak, K. A.; Flint, H. J.; Scott, K. P. Comparative analysis of sequences flanking tet $(\mathrm{W})$ resistance genes in multiple 
species of gut bacteria. Antimicrob. Agents Chemother. 2006, 50 (8), $2632-2639$.

(44) Eikmeyer, F.; Hadiati, A.; Szczepanowski, R.; Wibberg, D.; Schneiker-Bekel, S.; Rogers, L. M.; Brown, C. J.; Top, E. M.; Puhler, A.; Schluter, A. The complete genome sequences of four new IncN plasmids from wastewater treatment plant effluent provide new insights into IncN plasmid diversity and evolution. Plasmid 2012, 68 (1), 13-24.

(45) Coudron, P. E.; Moland, E. S.; Thomson, K. S. Occurrence and detection of AmpC beta-lactamases among Escherichia coli, Klebsiella pneumoniae, and Proteus mirabilis isolates at a veterans medical center. J. Clin. Microbiol. 2000, 38 (5), 1791-1796.

(46) Doublet, B.; Schwarz, S.; Kehrenberg, C.; Cloeckaert, A. Florfenicol resistance gene $f l o R$ is part of a novel transposon. Antimicrob. Agents Chemother. 2005, 49 (5), 2106-2108.

(47) Okitsu, N.; Kaieda, S.; Yano, H.; Nakano, R.; Hosaka, Y.; Okamoto, R.; Kobayashi, T.; Inoue, M. Characterization of ermB gene transposition by $\operatorname{Tn} 1545$ and $\operatorname{Tn} 917$ in macrolide-resistant Streptococcus pneumoniae isolates. J. Clin. Microbiol. 2005, 43 (1), 168-173.

(48) Jechalke, S.; Kopmann, C.; Rosendahl, I.; Groeneweg, J.; Weichelt, V.; Krö gerrecklenfort, E.; Brandes, N.; Nordwig, M.; Ding, G. C.; Siemens, J.; Heuer, H.; Smalla, K. Increased abundance and transferability of resistance genes after field application of manure from sulfadiazine-treated pigs. Appl. Environ. Microbiol. 2013, 79 (5), 17041711.

(49) Gullberg, E.; Cao, S.; Berg, O. G.; Ilbäck, C.; Sandegren, L.; Hughes, D.; Andersson, D. I. Selection of resistant bacteria at very low antibiotic concentrations. PloS Pathog. 2011, 7 (7), e1002158.

(50) Linares, J. F.; Gustafsson, I.; Baquero, F.; Martinez, J. L. Antibiotics as intermicrobial signaling agents instead of weapons. PNAS 2006, 103 (51), 19484-19489.

(51) Stanton, T. B.; Humphrey, S. B. Persistence of antibiotic resistance: Evaluation of a probiotic approach using antibiotic-sensitive megasphaera elsdenii strains to prevent colonization of swine by antibiotic-resistant strains. Applied. Environ. Microbiol. 2011, 77 (20), $7158-7166$.

(52) Looft, T.; Johnson, T. A.; Allen, H. K.; Bayles, D. O.; Alt, D. P.; Stedtfeld, R. D.; Sul, W. J.; Stedtfeld, T. M.; Chai, B.; Cole, J. R.; Hashsham, S. A.; Tiedje, J. M.; Stanton, T. B. In-feed antibiotic effects on the swine intestinal microbiome. PNAS 2012, 109 (5), 1691-1696. 